\title{
Quantum chemical studies on tuning the second-order nonlinear optical molecular switching of triarylborane derivatives
}

\author{
LI Xue, SUN ShiLing, MA NaNa, QIU YongQing* \& FU Qiang* \\ Institute of Functional Material Chemistry, Faculty of Chemistry, Northeast Normal University, Changchun 130024, China
}

Received August 10, 2011; accepted November 13, 2011; published online March 30, 2012

In this work, density functional theory (DFT) combined with the finite field (FF) method has been adopted to analyze the secondorder nonlinear optical (NLO) properties of the triarylborane (TAB) derivatives obtained by introducing different inductive electron groups into the phenylene ring of the TAB (RTAB, where $\left.\mathrm{R}=2-\mathrm{C}_{6} \mathrm{H}_{5}-\mathrm{C}_{2} \mathrm{~B}_{10} \mathrm{H}_{10}(\mathbf{1}), \mathrm{R}=\mathrm{F}(\mathbf{2}), \mathrm{R}=\mathrm{Me}(\mathbf{3}), \mathrm{R}=\mathrm{NO}_{2}(\mathbf{4}), \mathrm{R}=\mathrm{NH}_{2}(\mathbf{5})\right)$. The static first hyperpolarizabilities $\left(\beta_{\text {tot }}\right)$ of the RTAB molecules can be switched by binding one $\mathrm{F}^{-}$to the boron center (RTAB') or one-electron reduction (RTAB"). The DFT-FF calculations show that the $\beta_{\text {tot }}$ values of $\mathbf{2}^{\prime}, \mathbf{3}^{\prime}$ and $\mathbf{5}^{\prime}$ decrease while those of $\mathbf{1}^{\prime}$ and $\mathbf{4}^{\prime}$ increase compared with the values of their neutral molecules, which was attributed to the fact that the charge transfers of $\mathbf{2}$, $\mathbf{3}$ and $\mathbf{5}$ become smaller and those of $\mathbf{1}$ and $\mathbf{4}$ become larger by binding one $\mathrm{F}^{-}$ion to the boron center, according to time-domain DFT (TD-DFT) analysis. However, the incorporation of one electron enhances the second-order NLO properties of the RTAB molecules remarkably, especially for system $\mathbf{1}$. It is notable that the $\beta_{\text {tot }}$ value of reduced form 1 " is $508.69 \times 10^{-30}$ esu, i.e. about 578 times larger than that of system 1. Frontier molecular orbital (FMO) and natural bond orbital (NBO) analyses suggest that the reversal of the charge distribution between the neutral molecules and their reduced forms leads to low HOMO-LUMO energy gaps $\left(E_{0}\right)$ and thus large $\beta_{\text {tot }}$ values for the reduced forms.

triarylborane derivatives, second-order NLO property, NLO switching, DFT

Citation: $\quad$ Li X, Sun S L, Ma N N, et al. Quantum chemical studies on tuning the second-order nonlinear optical molecular switching of triarylborane derivatives. Chin Sci Bull, 2012, 57: 1772-1780, doi: 10.1007/s11434-012-5059-5

Novel molecular materials with optimal nonlinear optical (NLO) properties are still in great demand because of their primary roles in applications in fields including optical communications and computation, optical switching and limiting, data storage and retrieval, and sensors [1-4]. Recently, triarylborane $(\mathrm{TAB})$ and its derivatives have attracted increasing attention because of their strong photophysical properties, and have been making greater contributions in the fields of luminescence [5-8], optoelectronics (organic light-emitting diodes and electroluminescence (OLED/EL), nonlinear optical and two-photon absorption) [9-12], catalytic polymerization [13-15] and anion sensors [16-18], because their intriguing electronic structures come from the $\mathrm{p}_{\pi}-\pi$ conjugation through the vacant $\mathrm{p}$-orbital on the boron atom $(\mathrm{p}(\mathrm{B}))[19,20]$. It is well known that the molecular

*Corresponding authors (email: qiuyq466@nenu.edu.cn; fqiang@nenu.edu.cn) second-order NLO properties depend not only on the nature of the $\pi$-conjugation but also on the strengths of the donor and acceptor groups [21]. Thus, in this report, we have introduced the conventional electron donor or acceptor substituent groups to the phenylene ring of the TAB to optimize its NLO properties. We have also studied closo- $\mathrm{C}_{2} \mathrm{~B}_{10} \mathrm{H}_{12}$ carborane with an aryl group from the perspective of its use as a novel substituent on the phenylene ring of TAB. It was found that not only it does the carborane enhance Lewis acidity by the formation of an electron deficient cage, but also it leads to high stabilization of the vacant $\mathrm{p}(\mathrm{B})$ for the TAB used in the experiment [22].

Although efforts over several decades in the NLO field have resulted in far-ranging studies on the design, synthesis, structure, reactivity and application of these materials, a number of strategies have also been extensively studied as a part of this progress [23]. The primary aim is to be able to 
reversibly switch and effectively modulate the NLO response of a molecule [24,25]. To solve this problem, some novel concepts including oxidation/reduction [21], photoisomerization [26], photoinduced proton transfer [25], and protonation/deprotonation [24] have been extensively investigated experimentally and theoretically, particularly for the redox switching of NLO properties. Among the redox switching methods, Coe et al. [27,28] and Li et al. [29,30] reported that the second-order NLO properties of many new chromophores change substantially under the influence of these redox processes. We therefore also decided to investigate the effects of redox switching on the NLO properties of our systems.

It has also been reported that the boron (B) atom shows a strong affinity toward fluoride ion $\left(\mathrm{F}^{-}\right)$, and the binding of $\mathrm{F}^{-}$to the $\mathrm{B}$ center can switch and modulate the material's optical properties [31,32]. For example, the spectroscopic properties of a luminescent rhenium(I) tricarbonyl diimine complex with a TAB moiety and its $\mathrm{F}^{-}$derivative were investigated by density functional theory (DFT) approach, where binding of $\mathrm{F}^{-}$results in reconfiguration of the molecular geometry and variation of the optical properties [33]. The NLO properties of dibenzoborole derivatives were calculated by Muhammad et al. [34]. Their results show that the binding of one $\mathrm{F}^{-}$to the $\mathrm{B}$ atom of dibenzoborole or the one-electron reduction process leads to a higher charge transfer (CT), thus larger $\beta$ values should be obtained. This indicates that the NLO properties of TAB and its derivatives could be also modified by binding $\mathrm{F}^{-}$to the $\mathrm{B}$ center.

We have adopted quantum-chemical calculations to analyze the electronic structure, linear optical properties and NLO properties of a series of triarylborane derivatives in this work. To utilizeuse these molecules more effectively, we have analyzed NLO properties switching for these compounds by reducing one electron to neutral form and binding $\mathrm{F}^{-}$to a boron center.

\section{Theoretical methods and calculation models}

All calculations were performed using Gaussian $09 \mathrm{~W}$ program [35]. The geometries of all systems with all real frequencies were optimized using B3LYP level in combination with the $6-31 \mathrm{G}(\mathrm{d})$ basis set. The natural bond orbitals (NBOs) were calculated for the selected molecules at the same level. The molecular electronic spectra were obtained using TD-PBE0 (also known as PBE1PBE) exchange correlation functional with $6-31+\mathrm{G}(\mathrm{d})$ basis set. The PBE0 functional is based on the Perdew-Burke-Ernzerhof (PBE) generalized gradient functional by mixing of $25 \%$ of Hartree-Fock exchange, calculated using the Kohn-Sham orbital, with $75 \%$ of PBE exchange, and PBE0 method has been successfully adopted to study the electronic spectra of many systems [36-39].
In finite field (FF) method, when a molecule is subjected to a static electric field $(F)$, the energy $(E)$ of the molecule is expressed as

$$
\begin{aligned}
E= & E^{(0)}-\mu_{i} F_{i}-\frac{1}{2} \alpha_{i j} F_{i} F_{j}-\frac{1}{6} \beta_{i j k} F_{i} F_{j} F_{k} \\
& -\frac{1}{24} \gamma_{i j k l} F_{i} F_{j} F_{k} F_{l}-\cdots \cdots,
\end{aligned}
$$

where $E^{(0)}$ is the energy of the molecule in the absence of an electronic field, $\mu$ is a component of the dipole moment vector, $\alpha$ is the linear polarizability tensor, $\beta$ and $\gamma$ are the first and second hyperpolarizability tensors, and $i, j$ and $k$ are labels for the $x, y$, and $z$ components, respectively. For each molecule, the static first hyperpolarizability $\beta_{\text {tot }}$ was calculated using the following equation:

$$
\beta_{\mathrm{tot}}=\sqrt{\beta_{x}^{2}+\beta_{y}^{2}+\beta_{z}^{2}},
$$

$\beta_{x}, \beta_{y}$ and $\beta_{z}$ are the components of the second-order polarizability tensor along the $x$-, $y$ - and $z$-axes, respectively. They were calculated using the following equation:

$$
\beta_{i}=\beta_{i i i}+\frac{1}{3} \sum_{i \neq j}\left[\left(\beta_{i j j}+\beta_{j i j}+\beta_{i j i}\right)\right], \quad i, j=x, y, z .
$$

In this work, the first hyperpolarizabilities for all systems were evaluated using the FF approach with a field frequency of 0.0010 au at CAM-B3LYP/6-31+G(d) and BHandHLYP/ $6-31+\mathrm{G}(\mathrm{d})$ levels to check the consistency of our calculations. In many reports, it was found that CAM-B3LYP $[40,41]$ and BHandHLYP $[42,43]$ results are close to the reference CCSD and MP2 values, which indicates that both of these methods are very effective and accurate for calculation of the first hyperpolarizability. We have therefore adopted the two quantum chemical approaches to calculate the first hyperpolarizabilities of all systems studied.

As shown in Figure 1, the derivatives of TAB have been systematically investigated using three sets of chemical models in this work. Five neutral molecules, 1, 2, 3, 4 and 5, which are integrally called RTAB, are derived from TAB by introducing different inductive electron groups $(\mathrm{R})$ into the phenylene ring. The compounds of RTAB' $\left(\mathbf{1}^{\prime}, \mathbf{2}^{\prime}, \mathbf{3}^{\prime}, \mathbf{4}^{\prime}\right.$ and $\mathbf{5}^{\prime}$ ) are obtained by binding one $\mathrm{F}^{-}$to the $\mathrm{B}$ atom of the neutral molecules. RTAB" represents reduced forms of RTAB consisting of 1", 2", 3", 4" and 5". Among these models, 1, 2, 3 and 1' have been experimentally synthesized with good thermal stabilities by Huh et al. [22], while other compounds were designed to compare properties with system $\mathbf{1}$ in this study. The origin of the coordinate system is placed in the $\mathrm{C}(5)$ atom with the $z$-axis along the bonding orientation between the $\mathrm{C}(5)$ atom and the $\mathrm{B}(1)$ atom. The $x z$ plane is the plane of the phenylene (as seen in Figure 1).

The selected bond lengths and angles of systems $\mathbf{1}$ and $\mathbf{1}$ optimized with B3LYP/6-31G(d) method are listed in Table 1. The calculated data are seen to be in excellent agreement 


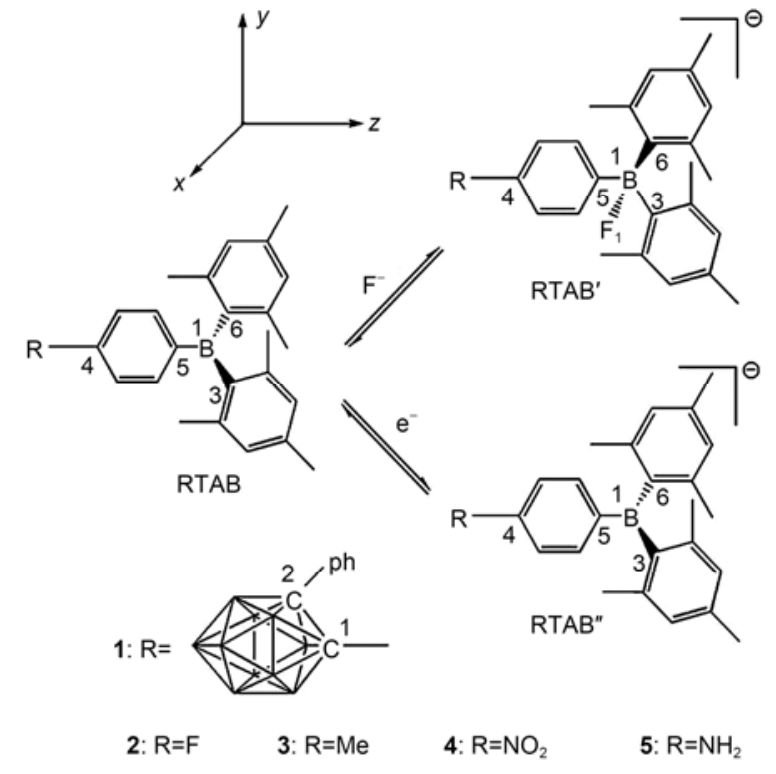

Figure 1 Representations of all adopted chemical models containing a $\mathrm{TAB}$ core.

with the experimental data, which suggests that the method and basis sets selected in this job are highly reliable for the systems studied.

\section{Results and discussion}

\subsection{Electronic spectrum}

The TD-DFT calculations were performed to obtain the electronic spectra of the closed-shell systems $\mathbf{1}-\mathbf{5}$ and $\mathbf{1}^{\prime}-\mathbf{5}^{\prime}$ at PBE1PBE/6-31+G(d) level. Table 2 shows the absorption wavelengths $(\lambda)$, transition energies $(E)$, oscillator strengths $(f)$ and major contributions of all of the closed-shell systems. The calculated results show that the strong absorption values of systems 1, $\mathbf{2}$ and $\mathbf{3}$ are at 272.5, 264.2 and $269.7 \mathrm{~nm}$, respectively, and these results are in good agreement with the experimental results reported by Lee et al. [22] $(270 \mathrm{~nm}$ for $\mathbf{1}, 260 \mathrm{~nm}$ for $\mathbf{2}$ and $265 \mathrm{~nm}$ for $\mathbf{3}$ ). This suggests that PBE1PBE/6-31+G(d) is a good choice to investigate the absorption spectra in our study. The electron density difference (EDD) maps involved in the crucial transitions are shown in Figure 2.

Obviously, from Figure 2, the absorption band of system $\mathbf{1}$ is assigned the crucial excitation that gives the CT from the phenylene moiety to $\mathrm{p}(\mathrm{B})$. Analogous electronic spectra are observed in systems $\mathbf{2}$ and $\mathbf{3}$, despite a slight blue-shift compared with $\mathbf{1}$, although the $\mathrm{R}$ group is different. In particular, the strong absorption of system $\mathbf{4}$ is mainly attributed to a crucial excited state containing a strong CT from the phenylene and dimesitylboron $\left(\mathrm{Mes}_{2}\right)$ to the $\mathrm{p}(\mathrm{B})$ and nitrogen, and the red-shift compared to 1-3 may arise from this CT pattern. For system 5, the electron transition consists of the $\mathrm{CT}$ from the amino group and phenylene to the vacant $\mathrm{p}(\mathrm{B})$ and the $\pi-\pi^{*}$ transition of conjugated phenylene, which leads to a further red-shift of the absorption band.

For the corresponding $\mathrm{F}^{-}$compounds, the crucial electronic transitions of $\mathbf{1}^{\prime}$ can be considered as a transition from $\mathrm{F}^{-}$, phenylene and $\mathrm{Mes}_{2}$ to the phenyl group linked with carborane. A stronger degree of CT than $\mathbf{1}$ is the main reason for the red-shift of $\mathbf{1}^{\prime}$. The absorptions of systems $\mathbf{2}^{\prime}$ and $3^{\prime}$ are similar because the crucial excited states both originate from the $\mathrm{Mes}_{2}$ to phenylene transition. Interestingly, the participation of $\mathrm{F}^{-}$is not found, which may be the reason why blue-shifts are observed in $\mathbf{2}^{\prime}$ and $\mathbf{3}^{\prime}$. The electronic spectrum of system $\mathbf{4}^{\prime}$ is similar to that of $\mathbf{1}^{\prime}$ because of the participation of $\mathrm{F}^{-}$beside the promotion of the electron from $\mathrm{Mes}_{2}$ to nitrogen, with a small contribution from phenylene, and the red-shift compared with the neutral system 4 is logical. Unexpectedly, for system $\mathbf{5}^{\prime}$, the crucial electronic transitions correspond to the CT from the amino group and phenylene to $\mathrm{Mes}_{2}$, and $\mathrm{F}^{-}$again does not take any part in the CT, similar to systems $\mathbf{2}^{\prime}$ and $\mathbf{3}^{\prime}$, and thus a blue-shift was observed. Careful analysis of the EDD maps for the neutral molecules and their $\mathrm{F}^{-}$compounds shows that in systems $\mathbf{1}, \mathbf{2}, \mathbf{3}, \mathbf{4}$ and $\mathbf{5}$, the $\mathrm{B}$ atom plays a major role in the formation of the unoccupied molecular orbital because of the electron deficient structure formed by the vacant $\mathrm{p}(\mathrm{B})$. The vacant $\mathrm{p}(\mathrm{B})$ effectively overlaps with the adjacent $\pi$-skeleton of the benzene rings and gathers most of the electrons to itself. However, the binding of $\mathrm{F}^{-}$to the $B$ atom turns off the $\mathrm{p}_{\pi}-\pi^{*}$ conjugation of vacant $\mathrm{p}(\mathrm{B})$ in the $\mathrm{F}^{-}$compounds, resulting in the large $\mathrm{CT}$ in systems $\mathbf{1}^{\prime}$ and $\mathbf{4}^{\prime}$, and the small CT in systems $\mathbf{2}^{\prime}, \mathbf{3}^{\prime}$ and $\mathbf{5}^{\prime}$. These changes

Table 1 The selected bond lengths ( $(\AA)$ and angles (deg) in systems 1, 1" and 1' with B3LYP/6-31G(d) method ${ }^{\text {a) }}$

\begin{tabular}{lccccccc}
\hline Bond length & $\mathbf{1}$ & $\mathbf{1}^{\prime \prime}$ & \multicolumn{1}{c}{$\mathbf{1}^{\prime}$} & Bond angle & $\mathbf{1}^{\prime}$ & $\mathbf{1}^{\prime \prime}$ & $\mathbf{1}^{\prime}$ \\
\hline $\mathrm{B}(1)-\mathrm{C}(6)$ & $1.582(1.578)$ & 1.598 & $1.6741(1.660)$ & $\mathrm{C}(5)-\mathrm{B}(1)-\mathrm{C}(3)$ & $118.641(119.940)$ & 119.528 & $106.436(105.323)$ \\
$\mathrm{B}(1)-\mathrm{C}(3)$ & $1.582(1.578)$ & 1.599 & $1.6716(1.648)$ & $\mathrm{C}(5)-\mathrm{B}(1)-\mathrm{C}(6)$ & $118.301(118.501)$ & 119.623 & $118.059(116.254)$ \\
$\mathrm{B}(1)-\mathrm{C}(5)$ & $1.577(1.571)$ & 1.543 & $1.656(1.641)$ & $\mathrm{C}(2)-\mathrm{C}(1)-\mathrm{C}(4)$ & $119.461(117.357)$ & 122.988 & $119.379(118.371)$ \\
$\mathrm{C}(4)-\mathrm{C}(1)$ & $1.510(1.506)$ & 1.456 & $1.496(1.506)$ & $\mathrm{F}(1)-\mathrm{B}(1)-\mathrm{C}(3)$ & - & - & $109.877(111.797)$ \\
$\mathrm{C}(1)-\mathrm{C}(2)$ & $1.758(1.722)$ & 2.356 & $1.805(1.723)$ & $\mathrm{F}(1)-\mathrm{B}(1)-\mathrm{C}(5)$ & - & - & $104.022(106.082)$ \\
$\mathrm{B}(1)-\mathrm{F}(1)$ & - & - & $1.458(1.485)$ & & & \\
\hline
\end{tabular}

a) Experimental values have been taken from ref. [22]. 
Table 2 TD-DFT calculations at PBE1PBE/6-31+G(d) level for different systems studied in RTAB and RTAB'

\begin{tabular}{|c|c|c|c|c|}
\hline Molecule & $\lambda(\mathrm{nm})$ & $E(\mathrm{eV})$ & Major contribution & $f$ \\
\hline 1 & 272.5 & 4.55 & $\begin{array}{l}\mathrm{HOMO}_{-4}-\mathrm{LUMO}(60 \%) \\
\mathrm{HOMO}_{-5}-\mathrm{LUMO}(32 \%)\end{array}$ & 0.2871 \\
\hline $\mathbf{1}^{\prime}$ & 326.5 & 3.80 & $\begin{array}{l}\mathrm{HOMO}_{-4}-\mathrm{LUMO}(63 \%) \\
\mathrm{HOMO}_{-3}-\mathrm{LUMO}(34 \%)\end{array}$ & 0.0593 \\
\hline 2 & 264.2 & 4.69 & $\mathrm{HOMO}_{-4}-\mathrm{LUMO}(93 \%)$ & 0.1630 \\
\hline $2^{\prime}$ & 239.2 & 5.18 & $\begin{array}{l}\mathrm{HOMO}_{-1}-\mathrm{LUMO}_{+2}(25 \%) \\
\mathrm{HOMO}_{-4}-\mathrm{LUMO}(14 \%)\end{array}$ & 0.0517 \\
\hline 3 & 269.7 & 4.60 & $\mathrm{HOMO}_{-4}-\mathrm{LUMO}(92 \%)$ & 0.1964 \\
\hline $3^{\prime}$ & 235.8 & 5.26 & $\mathrm{HOMO}_{-2}-\mathrm{LUMO}_{+3}(35 \%)$ & 0.0420 \\
\hline 4 & 286.8 & 4.32 & $\mathrm{HOMO}_{-4}-\mathrm{LUMO}_{+5}(33 \%)$ & 0.1785 \\
\hline $4^{\prime}$ & 348.2 & 3.56 & $\mathrm{HOMO}_{-4}-\mathrm{LUMO}(98 \%)$ & 0.2351 \\
\hline 5 & 324.6 & 3.82 & HOMO-LUMO(76\%) & 0.2800 \\
\hline $5^{\prime}$ & 253.9 & 4.88 & $\begin{array}{l}\mathrm{HOMO}_{\mathrm{LUMO}}^{+6}(34 \%) \\
\mathrm{HOMO}_{-\mathrm{LUMO}_{+7}(10 \%)}\end{array}$ & 0.0330 \\
\hline
\end{tabular}

have an important effect on the NLO responses of the $\mathrm{F}^{-}$ compounds.

\subsection{NLO properties of the studied systems}

The first hyperpolarizabilities for RTAB and RTAB' were evaluated by the FF approach using CAM-B3LYP/6-31+ $\mathrm{G}(\mathrm{d})$ and BHandHLYP/6-31+G(d) levels. The components $\beta_{x}, \beta_{y}, \beta_{z}$ and the static first hyperpolarizability $\beta_{\text {tot }}$ values for all of the systems are presented in Table 3. As shown in Table 3, although the $\beta_{\text {tot }}$ values obtained by CAM-B3LYP method are slightly larger than those obtained by BHandHLYP method, the overall change trend obtained by both methods is consistent, and thus the static first hyperpolarizabilities of RTAB" have been calculated at only CAM-B3LYP/6-31+ G(d) level, considering the larger size of the systems studied and the associated computational costs.

(1) Closed-shell systems 1-5 and 1'-5'. Some changes in the NLO properties can be observed after introducing

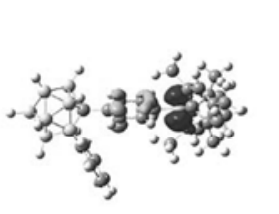

1

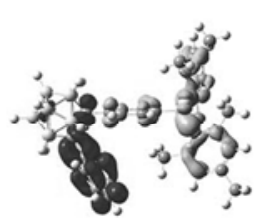

$1^{\prime}$

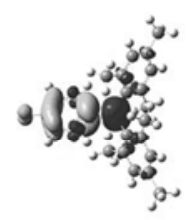

2

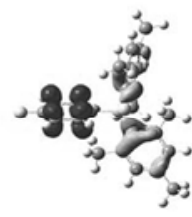

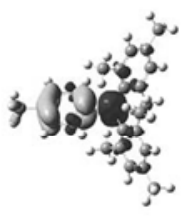

3

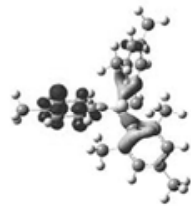

$3^{\prime}$

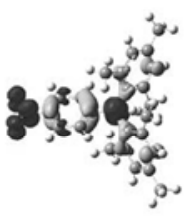

4

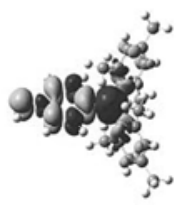

5
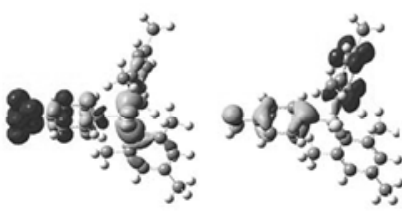

$5^{\prime}$

Figure 2 Electron density difference (EDD) maps in crucial electronic excitations for systems $\mathbf{1}^{-5}$ and $\mathbf{1}^{\prime}-\mathbf{5}^{\prime}$.

Table 3 Static first hyperpolarizabilities $\left(\times 10^{-30} \mathrm{esu}\right)$ of RTAB, RTAB' and RTAB" with CAM-B3LYP/6-31+G(d) and BHandHLYP/6-31+G(d) methods

\begin{tabular}{|c|c|c|c|c|c|c|c|c|}
\hline \multirow{2}{*}{ Molecule } & \multicolumn{2}{|c|}{$\beta_{x}$} & \multicolumn{2}{|c|}{$\beta_{y}$} & \multicolumn{2}{|c|}{$\beta_{z}$} & \multicolumn{2}{|c|}{$\beta_{\text {tot }}$} \\
\hline & BHandHLYP & CAM-B3LYP & BHandHLYP & CAM-B3LYP & BHandHLYP & CAM-B3LYP & BHandHLYP & CAM-B3LYP \\
\hline 1 & 0.28 & 0.26 & -0.51 & -0.24 & -0.43 & -0.81 & 0.57 & 0.88 \\
\hline $\mathbf{1}^{\prime}$ & -0.21 & -0.18 & 2.03 & 1.91 & -16.09 & -17.03 & 16.22 & 17.14 \\
\hline $\mathbf{1}^{\prime \prime}$ & - & 9.06 & - & -65.84 & - & 504.33 & - & 508.69 \\
\hline 2 & -4.21 & -0.45 & 0.23 & 0.24 & 3.84 & 4.10 & 3.87 & 4.13 \\
\hline $2^{\prime \prime}$ & - & -2.44 & - & -0.57 & - & 30.92 & - & 31.02 \\
\hline 3 & -0.03 & -0.13 & 0.37 & 0.32 & 4.19 & 4.20 & 4.19 & 4.21 \\
\hline $\mathbf{3}^{\prime}$ & -1.69 & -1.75 & 0.34 & 0.27 & -0.72 & -0.19 & 1.72 & 1.78 \\
\hline $3^{\prime \prime}$ & - & 0.15 & - & -1.21 & - & 3.54 & - & 3.74 \\
\hline 4 & 0.33 & 0.55 & -0.16 & -0.43 & -8.33 & -8.62 & 8.33 & 8.65 \\
\hline $4^{\prime}$ & 0.31 & 0.30 & -0.34 & -0.55 & -17.20 & -18.24 & 17.20 & 18.25 \\
\hline 5 & -0.72 & -0.76 & -0.43 & -0.42 & 14.34 & 15.03 & 14.37 & 15.05 \\
\hline $5^{\prime}$ & -2.38 & -2.48 & 0.82 & 0.74 & 3.68 & 3.83 & 4.45 & 4.62 \\
\hline $5 "$ & - & -2.19 & - & -2.56 & - & 34.72 & - & 34.88 \\
\hline
\end{tabular}


different inductive electron groups into TAB. The $\beta_{\text {tot }}$ values obtained are shown in Table 3 . For the neutral forms $\mathbf{1 - 5}$, the $\beta_{\text {tot }}$ value decreases in the order $\mathbf{5}>\mathbf{4}>\mathbf{2} \approx \mathbf{3}>\mathbf{1}$. For the various $\mathrm{R}$ groups, the amino group is a strong electron-donating group and the nitrogen is a strong electron acceptor. We concluded that the introduction of a strong electron-donating group into $\mathrm{TAB}$ would be favorable for improving NLO responses, while the fluorine and methyl act as donors according to the EDD maps. Unexpectedly, the NLO responses of $\mathbf{2}$ and $\mathbf{3}$ are found to be smaller than that of $\mathbf{4}$ with the electron acceptors, which implies that the CT pattern of $\mathbf{4}$ is polydirectional in comparison with the others. Actually, the carborane is sufficiently electron-deficient to be an electron acceptor, but no CT occurs on it, which may be attributed to the electron-rich phenyl group compromising the donor-acceptor ability. The CT of system $\mathbf{1}$ is thus weaker than that of the other systems, and causes the smallest NLO responses.

For the RTAB' compounds, when compared with the $\beta_{\text {tot }}$ of their respective neutral molecules, the values of $2^{\prime}, 3^{\prime}$ and $\mathbf{5}^{\prime}$ decrease, but those of $\mathbf{1}^{\prime}$ and $\mathbf{4}^{\prime}$ increase.

To further explain the origins of the second-order NLO properties of these systems, a widely used two-level model is applied to estimate $\beta$ :

$$
\beta \propto \frac{\Delta \mu \cdot f}{E^{3}},
$$

where $f, E$ and $\Delta \mu$ are the oscillator strength, the transition energy and the difference between the dipole moments of the ground state and the $m$ th excited state, respectively. In the two-level expression, the first hyperpolarizability, $\beta$, is proportional to the oscillator strength and is inversely proportional to the cube of the transition energy. For a molecule, the transition energy is therefore the decisive factor for large $\beta$ values.

As seen in Table 2, the transition energies $(E)$ are in the order of $\mathbf{2}>\mathbf{3}>\mathbf{4}>\mathbf{5}$, while the $\beta_{\mathrm{tot}}$ values of these systems are in the order of $\mathbf{5}>\mathbf{4}>\mathbf{3}>\mathbf{2}$. The $\beta_{\text {tot }}$ values are proportional to the $E$ values, which is in close agreement with the two-level model. The same phenomena are observed for both the $E$ values and the $\beta_{\text {tot }}$ values in systems $\mathbf{1}^{\prime}-\mathbf{5}^{\prime}$. For system $\mathbf{1}$, although its $E$ value is smaller than that of $\mathbf{2}$ and $\mathbf{3}$, which is inconsistent with their $\beta_{\text {tot }}$ values, careful analysis of the EDD maps involved in the crucial transitions suggests that the CT of $\mathbf{1}$ is not obvious in the transition process compared with that of 2-5 in Figure 2, and thus its $\beta_{\text {tot }}$ value is the smallest. There is also a contrary relationship between the $\beta_{\text {tot }}$ values and $E$ values for the neutral molecules and their $\mathrm{F}^{-}$compounds. For example, compared with the $E$ value $(4.32 \mathrm{eV})$ of system $\mathbf{4}$, the $E$ value of system $\mathbf{4}^{\prime}$ is $3.56 \mathrm{eV}$ in the crucial transition, so it is reasonable that the $\beta_{\text {tot }}$ value $\left(18.25 \times 10^{-30}\right.$ esu) of $\mathbf{4}^{\prime}$ is larger than that of system $4\left(8.65 \times 10^{-30} \mathrm{esu}\right)$, which is also in agreement with the conclusions obtained from the $\mathrm{CT}$ of the crucial excited state.
The frontier molecular orbitals (FMOs) of all of the systems are shown in Figure 3. From Figure 3, it is obvious that the highest occupied molecular orbitals (HOMOs) of the neutral molecules 1, 2, 3 and $\mathbf{4}$ delocalize primarily over the $\mathrm{Mes}_{2}$, whereas the lowest unoccupied molecular orbitals (LUMOs) localize largely on the $\mathrm{B}$ atom of the TAB moiety and the phenylene, with a small contribution from $\mathrm{R}$. The $\mathrm{Mes}_{2}$ thus acts as the donor, while the phenylene and B atom are considered to be the acceptors in these systems. The HOMO-LUMO electronic transitions can be attributed to CT from the $\mathrm{Mes}_{2}$ to the phenylene and the vacant $\mathrm{p}(\mathrm{B})$. For their $\mathrm{F}^{-}$compounds, the HOMOs are primarily centered on the $\mathrm{Mes}_{2}$ and sparingly centered on the $\mathrm{B}$ atom and $\mathrm{F}^{-}$, while the LUMOs are found on the phenylene and R. The HOMO-LUMO electronic transitions correspond to CT from the $\mathrm{Mes}_{2}$ and $\mathrm{p}(\mathrm{B})$ to phenylene and the $\mathrm{R}$ groups in these systems. Also, the CT in systems $\mathbf{2}^{\prime}$ and $\mathbf{3}^{\prime}$ becomes smaller than that in their neutral forms, whereas that of $\mathbf{1}^{\prime}$ and 4' becomes greater than that of the neutral forms. These changes are in accordance with their $\beta_{\text {tot }}$ values. However, $\mathbf{5}$ and $\mathbf{5}^{\prime}$ are different from other systems in the same series. Their HOMOs are dominated by the amino group and phenylene, while the LUMOs are dominated by the $\mathrm{Mes}_{2}$ and the vacant $\mathrm{p}(\mathrm{B})$. Their HOMO-LUMO electronic transitions can be considered to be from the amino group and phenylene to $\mathrm{Mes}_{2}$ and the vacant $\mathrm{p}(\mathrm{B})$. The CT of $\mathbf{5}$ is smaller than that of 5'. Careful analysis of the FMOs for the neutral molecules and their $\mathrm{F}^{-}$compounds shows that in systems $\mathbf{1}$, 2,3 and 4, the B atom plays a major role in the formation of the LUMOs because of its electron deficient structure formed by the vacant $\mathrm{p}(\mathrm{B})$. The vacant $\mathrm{p}(\mathrm{B})$ effectively overlaps with the adjacent $\pi$-skeleton of the benzene rings and gathers most of the electrons. However, the binding of $\mathrm{F}^{-}$to the $\mathrm{B}$ atom turns off the $\mathrm{p}_{\pi^{-}} \pi^{*}$ conjugation of the vacant $\mathrm{p}(\mathrm{B})$ in their $\mathrm{F}^{-}$compounds, resulting in changes in the $\mathrm{CT}$, and thus in the various $\beta_{\mathrm{tot}}$ values. For the HOMOs, the vacant $\mathrm{p}(\mathrm{B}) \mathrm{s}$ of all $\mathrm{F}^{-}$compounds are occupied by binding one $\mathrm{F}^{-}$, which is different from the neutral molecules.

(2) Open-shell systems 1"-5". We first analyze the redox properties of RTAB". It is well known that changes in the electronic properties related to the HOMO and LUMO of a molecule can lead to a change in the redox properties. The distribution of the FMOs can thus be used to illustrate the redox properties of the molecules. As shown in Figure 3, the LUMO of system 1 localizes largely on the phenylene, $\mathrm{B}$ atom and carborane with phenyl group. It is thus likely that the phenylene, B atom and carborane with phenyl group are the reduction centers of $\mathbf{1}$. To prove this, spin density distribution (SDD) plots of all of the reduced systems are shown in Figure 3. We can see that $74.2 \%$ of the spin density of system 1" localizes on the carborane with phenyl group (Table 4), while the phenylene and B atom carry a smaller spin density, indicating that the carborane with phenyl group is the main reduced center in system 1. The 


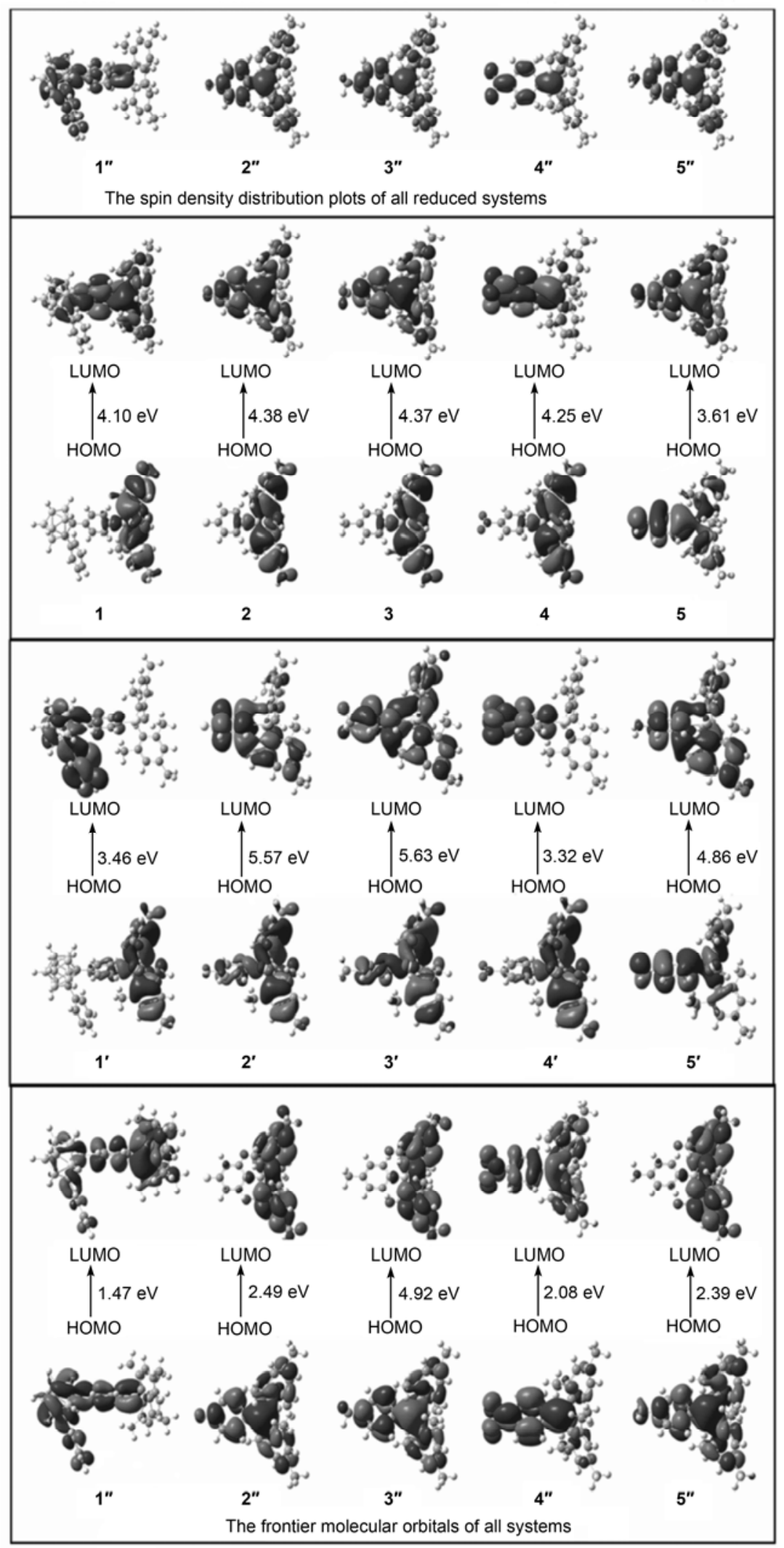

Figure 3 The frontier molecular orbitals (FMOs) of all systems and the spin density distribution (SDD) plots of all reduced systems.

geometric structure also shows a great change in the reduction center because of the incorporation of one electron.
Table 1 shows the key bond lengths and angles of $\mathbf{1}$ and $\mathbf{1}$ ", in which the $\mathrm{B}(1)-\mathrm{C}(6)$ and $\mathrm{B}(1)-\mathrm{C}(3)$ bond lengths are 
Table 4 The selected spin density distributions (SDD) of all reduced systems

\begin{tabular}{ccccc}
\hline Molecule & \multicolumn{1}{c}{$R$} & Phenylene & The boron atom & Dimesitylboron \\
\hline 1" & 0.742 & 0.152 & 0.138 & -0.032 \\
2" & 0.002 & 0.209 & 0.698 & 0.091 \\
3" & -0.001 & 0.231 & 0.685 & 0.085 \\
4" & 0.354 & 0.307 & 0.386 & -0.046 \\
5" & 0.016 & 0.156 & 0.693 & 0.134 \\
\hline
\end{tabular}

altered slightly, whereas the $\mathrm{C}(4)-\mathrm{C}(1)$ and $\mathrm{C}(1)-\mathrm{C}(2)$ bond lengths increase greatly during the reduction process. Similar changes in the corresponding bond angles are also observed. These bond parameters also indicate that reduction occurs mainly in the carborane with phenyl group for $\mathbf{1}$. The other four systems are quite different to system 1. The FMOs and the SDD plots show that the reduction centers of 2, $\mathbf{3}$ and $\mathbf{5}$ concentrate mostly on the B atom at about $70 \%$. The SDD plot of 4" localizes largely on the B atom at about $39 \%$, the phenylene at about $31 \%$ and the nitrogen at about $35 \%$, which is same as the LUMO distribution of 4 . Thus, the $\mathrm{B}$ atom, nitrogen and phenylene are the reduction centers for 4. In summary, the carborane with phenyl group has a greater ability to capture an extra electron than the other inductive electron groups involved in the reduction process.

We can see from Table 3 that the reduced forms 1", 2", $3^{\prime \prime}, \mathbf{4}^{\prime \prime}$ and 5" show significantly large $\beta_{\text {tot }}$ values of $508.69 \times$ $10^{-30}, 31.02 \times 10^{-30}, 3.47 \times 10^{-30}, 34.88 \times 10^{-30}$ and $197.26 \times$ $10^{-30}$ esu, respectively. Also, the $\beta_{\text {tot }}$ values of $\mathbf{1}^{\prime \prime}, \mathbf{2}^{\prime \prime}, \mathbf{4}^{\prime \prime}$ and 5" are 578, 7.51, 2.32 and 22.80 times larger than those of their corresponding neutral molecules, and only the $\beta_{\text {tot }}$ value of $\mathbf{3}^{\prime \prime}$ is smaller than that of $\mathbf{3}$; the reason for this is given in the following section. This indicates that the appropriate incorporation of one electron could cause a remarkable enhancement in the molecular second-order NLO properties.
The FMOs of all of the systems were analyzed to explain the origins of the unusual second-order NLO properties of the reduced forms (as seen in Figure 3). The HOMOs of the reduced forms have major contributions on the $\mathrm{R}$, phenylene and $\mathrm{B}$ atom, while the LUMOs mainly localize on the $\mathrm{Mes}_{2}$. The HOMO-LUMO electronic transitions can be attributed to CT from $\mathrm{p}(\mathrm{B}), \mathrm{R}$ and phenylene to $\mathrm{Mes}_{2}$. The $\mathrm{p}_{\pi}-\pi$ conjugation of vacant $\mathrm{p}(\mathrm{B})$ is turned off in the transition process. Consequently, $\mathrm{R}$ and phenylene act as electron donors, while $\mathrm{Mes}_{2}$ acts as an electron acceptor in the reduced forms. Alternatively, in the neutral systems 1, 2, 3 and 4, the $\mathrm{Mes}_{2}$ acts as the donor, and the phenylene and $\mathrm{B}$ atom are considered to be the acceptors. The HOMOLUMO electronic transitions correspond to CT from the $\mathrm{Mes}_{2}$ to phenylene and vacant $\mathrm{p}(\mathrm{B})$. This formation is regarded as a reversal of the charge distribution between the neutral molecules and their reduced systems, which leads to the low HOMO-LUMO energy gaps $\left(E_{0}\right)$ in the majority of the reduced systems, with the exception of $\mathbf{3}^{\prime \prime}$ (as seen in Figure 3). For system 1, the $E_{0}$ value of 1 is $4.10 \mathrm{eV}$, while that of $\mathbf{1}^{\prime \prime}$ is $1.47 \mathrm{eV}$. The smaller $E_{0}$ value causes a larger contribution to the second-order NLO properties, which is one of the crucial factors in explaining the larger $\beta_{\text {tot }}$ values of the reduced systems. However, for system $\mathbf{3}$, the electron reduction has no impact on the enhancement of the $\beta_{\text {tot }}$ value because of the small change to the $E_{0}$ value.

To shed further light on the reversal of the charge distribution, it is necessary to analyze the relationships of the electronic structure properties. NBO analyses of the partial neutral molecules and their reduced forms have therefore been performed. The combination of the results from the NBO analyses and the FMOs shows that system $\mathbf{1}$ has a small NBO charge $(-0.30234$ e) in the carborane with phenyl group and phenylene (as seen in Figure 4), and thus the carborane with phenyl group and the phenylene act as the weak acceptors and the $\mathrm{Mes}_{2}$ acts as the weak donor in the HOMO-LUMO electronic transitions (see Figure 3), which

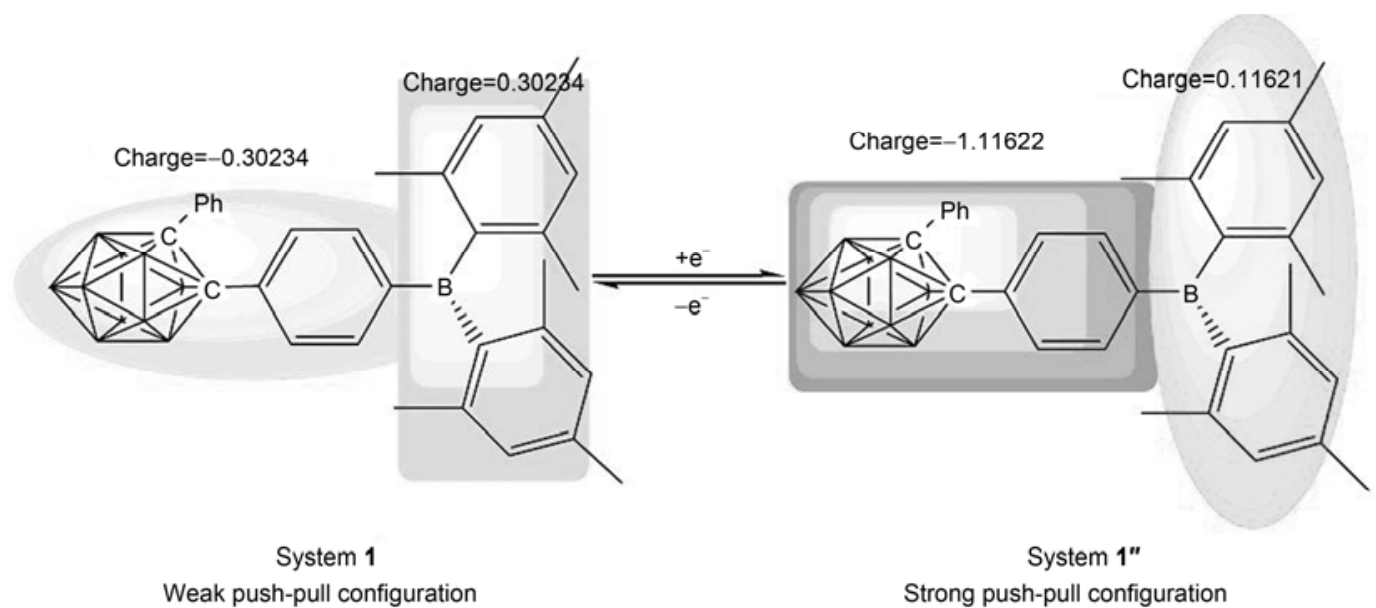

Figure 4 The NBO charges along with a graphical representation of systems $\mathbf{1}$ and $\mathbf{1}$ " explaining the reversal of charge distribution in the reduction process. 
indicates that system $\mathbf{1}$ has a weak push-pull configuration. For the reduced form of system $\mathbf{1}$ (system 1"), the negative charge is mainly distributed in the carborane with phenyl group and the phenylene $(-1.12 \mathrm{e})$. The carborane with phenyl group and the phenylene change into stronger donors because of the incorporation of the single electron in the transition process. This strong push-pull configuration helps to increase the NLO responses, and thus the second-order NLO properties of system 1" are the most outstanding. The reversals of the charge distribution for the other systems are similar. The NBO charge of the $\mathrm{R}$ group and phenylene is 0.32 e in system $\mathbf{4}$, while it is -0.89 e in system 4". We can see that the reversal of the charge distribution is the most noticeable between systems $\mathbf{1}$ and 1", leading to the greatest change in their second-order NLO properties.

In general, we can see that the changes in the $\beta_{\text {tot }}$ values are larger in the reduced forms than that in the $\mathrm{F}^{-}$compounds, especially for system 1 . Compared with the neutral form $\mathbf{1}$, the $\beta_{\text {tot }}$ value of the reduced form $\mathbf{1}^{\prime \prime}$ is 578 -fold larger, while the $\beta_{\text {tot }}$ value of the $\mathrm{F}^{-}$compound is 19.5 -fold larger. The substantial enhancement of the second-order NLO responses makes system $\mathbf{1}$ a potential candidate for a new type of "on/off" switchable NLO molecular material, where the neutral molecule $\mathbf{1}$ and $\mathrm{F}^{-}$compound $\mathbf{1}^{\prime}$ act as the trigger for the nonlinear switch-OFF, while the reduced anion 1" acts as the nonlinear switch-ON.

\section{Conclusions}

A systematic DFT calculation was carried out on a series of TAB derivatives. The DFT calculations show that the order of the $\beta_{\text {tot }}$ values for the molecules obtained by introducing different inductive electron groups into the phenylene ring of the TAB $(\mathrm{RTAB})$ is $\mathbf{5}>\mathbf{4}>\mathbf{2} \approx \mathbf{3}>\mathbf{1}$, which indicates that the introduction of a strong electron donor group into TAB could lead to very favorable improvements in the NLO properties. We have also looked at ways to reversibly switch and effectively modulate the NLO properties of the RTAB molecules by binding one $\mathrm{F}^{-}$ion to the boron center $\left(\mathrm{RTAB}^{\prime}\right)$ or reducing one electron (RTAB"). For the RTAB' compounds, the $\beta_{\mathrm{tot}}$ values of $\mathbf{2}^{\prime}, \mathbf{3}^{\prime}$ and $\mathbf{5}^{\prime}$ are smaller, but those of $\mathbf{1}^{\prime}$ and $\mathbf{4}^{\prime}$ are significantly larger than the values of their neutral forms because of the binding of the $\mathrm{F}^{-}$ion to the boron center. TD-DFT results and EDD map analyses of the RTAB and RTAB' molecules show that the binding of $\mathrm{F}^{-}$to the boron center causes a series of results, such as the red-shift in the absorption for $\mathbf{1}^{\prime}$ and $\mathbf{4}^{\prime}$, the blue-shift in the absorption for $\mathbf{2}^{\prime}, \mathbf{3}^{\prime}$ and $\mathbf{5}^{\prime}$, and the turning off of the $\mathrm{p}_{\pi^{-}} \pi^{*}$ conjugation of the vacant p-orbital of the boron atom. These results lead to a large charge transfer in systems $\mathbf{1}^{\prime}$ and $\mathbf{4}^{\prime}$, and smaller charge transfer in systems $2^{\prime}, 3^{\prime}$ and $5^{\prime}$. These changes are in accordance with their $\beta_{\text {tot }}$ values.

In the RTAB" forms, incorporating one extra electron in systems 1, 2, 4 and 5 causes significant enhancement of the second-order NLO responses, especially for system $\mathbf{1}$. The $\beta_{\text {tot }}$ value of system 1" is about 578 times as large as that of system 1. Compared with the neutral molecules, the charge distribution is reversed for the reduced forms, which leads to low HOMO-LUMO energy gaps and large $\beta_{\text {tot }}$ values for 1", 2", 4" and 5". For system 3, electron reduction has no impact on enhancement of the $\beta_{\text {tot }}$ value. FMO and NBO analyses show that system $\mathbf{1}$ has a weak push-pull configuration, while the push-pull configuration of system $\mathbf{1}^{\prime \prime}$ is strong, resulting in the lowest HOMO-LUMO energy gap and the largest $\beta$ value. Our investigation has not only confirmed the origins of these second-order NLO responses for the $\mathrm{F}^{-}$compounds and one-electron reductions of TAB derivatives, but has also predicted that the present boron systems can potentially provide significant "on/off" switchable NLO molecular materials.

This work was supported by the National Natural Science Foundation of China (20873017) and the Natural Science Foundation of Jilin Province (20101154).

1 Yang G C, Fang L, Tan K, et al. Quantum chemical study of structures, electronic spectrum, and nonlinear optical properties of goldpentacene complexes. Organomet, 2007, 26: 2082-2087

2 Allis D G, Spencer J T. Polyhedral-based nonlinear optical Materials. 2. Theoretical investigation of some new high nonlinear optical response compounds involving polyhedral bridges with charged aromatic donors and acceptors. Inorg Chem, 2001, 40: 3373-3380

3 Cheng W D, Xiang K H, Pandey R, et al. Calculations of linear and nonlinear optical properties of H-silsesquioxanes. J Phys Chem B, 2000, 104: 6737-6742

4 Ma N N, Qiu Y Q, Sun S L, et al. Theoretical investigation on structures, electronic spectra and nonlinear optical properties of gold compounds [X-\{Au( $\left.\left.\left.\mathrm{PMe}_{3}\right)\right\}_{2}\right]$. Sci China Chem, 2010, 53: 11491154

5 Parab K, Venkatasubbaiah K, Jäkle F. Luminescent triarylboranefunctionalized polystyrene: Synthesis, photophysical characterization, and anion-binding studies. J Am Chem Soc, 2006, 128: 12879-12885

6 Lam S T, Zhu N Y, Yam V W W. Synthesis and characterization of luminescent rhenium(I) tricarbonyl diimine complexes with a triarylboron moiety and the study of their fluoride ion-binding properties. Inorg Chem, 2009, 48: 9664-9670

7 Zhao Q, Li F Y, Liu S J, et al. Highly selective phosphorescent chemosensor for fluoride based on an iridium(III) complex containing arylborane units. Inorg Chem, 2008, 47: 9256-9264

8 Sakuda E, Tsuge K, Sasaki Y, et al. Spectroscopic and excited-state properties of tri-9-anthrylborane III: Crystal and spectroscopic polymorphisms. J Phys Chem B, 2005, 109: 22326-22331

9 Entwistle C D, Marder T B. Applications of three-coordinate organoboron compounds and polymers in optoelectronics. Chem Mater, 2004, 16: 4574-4585

10 Liu Y, Xuan W M, Zhang H, et al. Chirality- and threefold-symmetrydirected assembly of homochiral octupolar metal-organoboron frameworks. Inorg Chem, 2009, 48: 10018-10023

11 Yuan Z, Taylor N J, Sun Y, et al. Synthesis and second-order nonlinear optical properties of three coordinate organoboranes with diphenylphosphino and ferrocenyl groups as electron donors: Crystal and molecular structures of $(E)-\mathrm{D}-\mathrm{CH}=\mathrm{CH}-\mathrm{B}$ (mes) $)_{2}$ and $\mathrm{D}-\mathrm{C} \equiv \mathrm{C}-\mathrm{B}$ $(m e s)_{2}\left[\mathrm{D}=\mathrm{P}\left(\mathrm{C}_{6} \mathrm{H}_{52},\left(\eta-\mathrm{C}_{5} \mathrm{H}_{5}\right) \mathrm{Fe}\left(\mathrm{h}-\mathrm{C}_{5} \mathrm{H}_{4}\right)\right.\right.$; mes=2,4,6- $\left.\left(\mathrm{CH}_{3}\right)_{3} \mathrm{C}_{6} \mathrm{H}_{2}\right] . \mathrm{J}$ Organomet Chem, 1993, 449: 27-37

12 Yuan Z, Taylor N J, Marder T B, et al. Three coordinate phosphorus 
and boron as $\pi$-donor and p-acceptor moieties respectively, in conjugated organic molecules for nonlinear optics: Crystal and molecular structures of $E$ - $\mathrm{Ph}-\mathrm{CH}=\mathrm{CH}-\mathrm{B}(\mathrm{mes})_{2}, E-4-\mathrm{MeO}-\mathrm{C}_{6} \mathrm{H}_{4}-\mathrm{CH}=\mathrm{CH}-\mathrm{B}(\mathrm{mes})_{2}$, and $E-\mathrm{Ph}_{2} \mathrm{P}-\mathrm{CH}=\mathrm{CH}-\mathrm{B}(\mathrm{mes})_{2} \quad\left[\mathrm{mes}=2,4,6-\mathrm{Me}_{3} \mathrm{C}_{6} \mathrm{H}_{2}\right]$. J Chem Soc, Chem Commun, 1990, 1489-1492

13 Erker G. Tris(pentafluorophenyl)borane: A special boron Lewis acid for special reactions. Dalton Trans, 2005, 1883-1890

14 Piers W E. The chemistry of perfluoroaryl boranes. Adv Organomet Chem, 2004, 52: 1-76

15 Chen E Y X, Marks T J. Cocatalysts for metal-catalyzed olefin polymerization: Activators, activation processes, and structure-activity relationships. Chem Rev, 2000, 100: 1391-1434

16 Kubo Y, Yamamoto M, Ikeda M, et al. A colorimetric and ratiometric fluorescent chemosensor with three emission changes: Fluoride ion sensing by a triarylborane-porphyrin conjugate. Angew Chem Int Ed, 2003, 42: 2036-2040

17 Solé S, Gabbaï F P. A bidentate borane as colorimetric fluoride ion sensor. Chem Commun, 2004, 10: 1284-1285

18 Yamaguchi S, Akiyama S, Tamao K. Colorimetric fluoride ion sensing by boron-containing $\pi$-electron systems. J Am Chem Soc, 2001, 123: $11372-11375$

19 Sakuda E, Ando Y, Ito A, et al. Extremely large dipole moment in the excited singlet state of tris $\{[\mathrm{p}-(\mathrm{N}, N$-dimethylamino $)$ phenylethynyl $]$ duryl \}borane. J Phys Chem A, 2010, 114: 9144-9150

20 Sun Y, Wang S. Extending $\pi$-conjugation of triarylborons with a 2,2Bpy core: Impact of donor-acceptor geometry on luminescence, anion sensing, and metal ion binding. Inorg Chem, 2010, 49: 4394-4404

21 Liu C G, Guan W, Song P, et al. Redox-switchable second-order nonlinear optical responses of push-pull monotetrathiafulvalene-metalloporphyrins. Inorg Chem, 2009, 48: 6548-6554

22 Huh J O, Kim H, Lee K M, et al. $o$-Carborane-assisted Lewis acidity enhancement of triarylboranes. Chem Commun, 2010, 46: 1138-1140

23 Muhammad S, Xu H L, Liao Y, et al. Quantum mechanical design and structure of the $\mathrm{Li} @ \mathrm{~B}_{10} \mathrm{H}_{14}$ basket with a remarkably enhanced electro-optical response. J Am Chem Soc, 2009, 131: 11833-11840

24 Costes J P, Lamere J F, Lepetit C, et al. Synthesis, crystal structures, and nonlinear optical (NLO) properties of new schiff-base nickel(II) complexes. Toward a new type of molecular switch? Inorg Chem, 2005, 44: 1973-1982

25 Houbrechts S, Clays K, Persoons A, et al. Hyper-Rayleigh scattering investigation of nitrobenzyl pyridine model compounds for optical modulation of the hyperpolarisability. Chem Phys Lett, 1996, 258: 485-489

26 Viau L, Bidault S, Maury O, et al. All-optical orientation of photoisomerizable octupolar zinc(II) complexes in polymer films. J Am Chem Soc, 2004, 126: 8386-8387

27 Coe B J. Switchable nonlinear optical metallochromophores with pyridinium electron acceptor groups. Acc Chem Res, 2006, 39: 383393

28 Coe B J, Fielden J, Foxon S P, et al. Diquat derivatives: Highly active, two-dimensional nonlinear optical chromophores with potential redox switchability. J Am Chem Soc, 2010, 132: 10498-10512
29 Chen W, Li Z R, Wu D, et al. The structure and the large nonlinear optical properties of Li@Calix[4]pyrrole. J Am Chem Soc, 2005, 127: 10977-10981

30 Wang F F, Li Z R, Wu D, et al. Structures and considerable static first hyperpolarizabilities: New organic alkalides $\left(\mathrm{M}^{+} @ n^{6} \mathrm{adz}\right) \mathrm{M}^{\prime-}(\mathrm{M}$, $\left.\mathrm{M}^{\prime}=\mathrm{Li}, \mathrm{Na}, \mathrm{K} ; n=2,3\right)$ with cation inside and anion outside of the cage complexants. J Phys Chem B, 2008, 112: 1090-1094

31 Li J, Zhang G X, Zhang D Q, et al. Boron-containing monopyrroloannelated tetra thiafulvalene compounds: Synthesis and absorption spectral/electrochemical responsiveness toward fluoride ion. J Org Chem, 2010, 75: 5330-5333

32 Muhammad S, Xu H L, Janjua M R S A, et al. Quantum chemical study of benzimidazole derivatives to tune the second-order nonlinear optical molecular switching by proton abstraction. Phys Chem Chem Phys, 2010, 12: 4791-4799

33 Wang J, Bai F Q, Xia B H, et al. A DFT exploration of luminescent rhenium(I) tricarbonyl diimine complex with a triarylboron moiety and its F derivative. J Organomet Chem, 2011, 696: 2943-2948

34 Muhammad S, Janjua M R S A, Su Z M. Investigation of dibenzoboroles having $\pi$-electrons: Toward a new type of two-dimensional NLO molecular switch? J Phys Chem C, 2009, 113: 12551-12557

35 Frisch M J, Trucks G W, Schlegel H B, et al. Gaussian 09 User's Reference, 2009

36 Adamo C, Scuseria G E, Barone V. Accurate excitation energies from time-dependent density functional theory: Assessing the PBE0 model. J Chem Phys, 1999, 111: 2889-2899

37 Zhang T T, Jia J F, Ren Y, et al. Ligand effects on structures and spectroscopic properties of pyridine-2-aldoxime complexes of $\operatorname{Re}(\mathrm{CO})_{3}^{+}$: DFT/TDDFT theoretical studies. J Phys Chem A, 2011, 115: 31743181

38 Dumur F, Mayer C R, Hoang-Thi K, et al. Electrochemical, linear optical, and nonlinear optical properties and interpretation by density functional theory calculations of (4- $N, N$-dimethylaminostyryl)-pyridinium pendant group associated with polypyridinic ligands and respective multifunctional metal complexes $\left(\mathrm{Ru}^{\mathrm{II}}\right.$ or $\left.\mathrm{Zn}^{\mathrm{II}}\right)$. Inorg Chem, 2009, 48: 8120-8133

39 Adamo C, Barone V. Toward reliable density functional methods without adjustable parameters: The PBE0 model. J Chem Phys, 1999, 110: 6158-6170

40 Yanai T, Tew D P, Handy N C. A new hybrid exchange-correlation functional using the Coulomb-attenuating method (CAM-B3LYP). Chem Phys Lett, 2004, 393: 51-57

41 Lu S I. Computational study of static first hyperpolarizability of donor-acceptor substituted $(E)$-benzaldehyde phenylhydrazone. J Comput Chem, 2011, 32: 730-736

42 Hu Y Y, Sun S L, Muhammad S, et al. How the number and location of lithium atoms affect the first hyperpolarizability of grapheme. J Phys Chem C, 2010, 114: 19792-19798

43 Zhao H B, Qiu Y Q, Liu C G, et al. Redox-switchable second-order nonlinear optical responses of TEMPO-dithiolate ligand and (tempodt)M complexes ( $\mathrm{M}=\mathrm{Pt}, \mathrm{Pd})$. J Organomet Chem, 2010, 695: 2251-2257

Open Access This article is distributed under the terms of the Creative Commons Attribution License which permits any use, distribution, and reproduction in any medium, provided the original author(s) and source are credited. 